\title{
EFECTO DEL FUEGO SOBRE LA FAUNA DE INVERTEBRADOS DE UN PASTIZAL AL NOROESTE DE CORRIENTES
}

\author{
Armúa de Reyes, Cristina ${ }^{1}$; Bernardis, Aldo C ${ }^{2}$; Mazza, Silvia M. ${ }^{2}$; Goldfarb, María C. ${ }^{3}$ \\ 1-Facultad de Ciencias Exactas y Naturales y Agrimensura UNNE 2- Facultad de Ciencias Agrarias
} (UNNE) 3- INTA EEA. Corrientes.

\begin{abstract}
RESUMEN
Con el fin de evaluar el efecto del fuego sobre la fauna de invertebrados en un pastizal con predominio de Andropogon lateris $\mathrm{y}$ Sorghastrum agrostoides, en el Noroeste de la provincia de Corrientes, Argentina, se realizaron relevamientos y descripción de su comportamiento pre y post quema en el área de estudio. Entre 1998 y 2000, se aplicaron al pastizal diferentes frecuencias de quema, en un diseño en bloques al azar con tres repeticiones, evaluándose periódicamente la cantidad y diversidad de la fauna de invertebrados. Se realizó análisis multivariante de conglomerados jerárquicos, con distancia euclídea, para evaluar el comportamiento de la fauna estudiada por oportunidad de muestreo. Se realizó análisis de la variancia por grupo taxonómico y posteriormente la prueba de Tukey para separación de medias. Tanto en los muestreos de prequema como los de postquema los grupos de invertebrados presentes fueron en su mayoría de las clases: Insecta, Arachnida (Aranea y Acarina), Crustacea, Miriápoda, Annelida, Nemátoda y Molusca.
\end{abstract}

Palabras Clave: Quema, Insecta, Andropogon lateralis, Sorghartrum agrostoides.

\section{SUMMARY}

With the object of evaluate the burning effect on the invertebrate fauna an Andropogon lateralis and Sorghastrum agrostoides grassland located at the northwest of the Corrientes province, Argentina, was relieved and description of the behavior before and after burns in the study area were done. Between 1998 and 2000, different burns frequencies were applied to the grassland in a randomized block design with three replications and invertebrate fauna quantity and diversity were evaluated periodically. Multivariate analysis (hierarchical) conglomerate with euclidean distance) was used to evaluate fauna behavior at each sampling period. For each taxonomic groups an ANOVA was perfomed and Tukey's mean separation procedure used. At the preburning as well as the post-burning sampling the invertebrate groups presents belonged to the arthropoda phylum: Insecta, Arachnida (Aranea and Acarina), Crustacea, Miriapoda, Annelida, Nematoda and Molusca.
Key words: Burns, Andropogon lateralis, Sorghastrum agrostoides.

\section{INTRODUCCION}

El fuego es una perturbación de reconocida incidencia en los ecosistemas, determinante de muchas características estructurales y funcionales de las comunidades (Mooney \& Hobbs, 1986). Características como el rebrote o la germinación después de la quema son adaptaciones funcionales a este tipo de perturbación, especialmente cuando los fuegos se manifiestan como perturbaciones recurrentes (Vera et al, 1995).

Desde el punto de vista ecológico, el fuego es algo más que una reacción química, es un fenómeno de primera magnitud, generalmente responsable de la evolución de gran cantidad de ecosistemas terrestres. Es la causa de que muchas praderas, sabanas y matorrales no evolucionen nunca hasta el climax de bosque o hasta el climax climático que les correspondería (GarcíaVillanueva, 1992).

Si bien el fuego es un fenómeno natural vinculado a la dinámica de muchas formaciones vegetales, su efecto, tanto sobre la evolución de la vegetación como en la producción secundaria lograda en los ecosistemas, es escasamente conocido. Tárrega (1986) señala que el efecto del fuego sobre los pastizales depende de la intensidad, la estación y la frecuencia de las quemas.

En la mayoría de los casos el fuego ejerce una acción positiva sobre la comunidad de pastizales, ya que favorece el rebrote y mejora la calidad de los mismos. No ocurre esto respecto a la fauna entomológica aérea que necesita de sus huéspedes para alimentarse, oviponer, cumplimentar su ciclo biológico y perpetuar la especie

En la Provincia de Corrientes, según Carnevalli (1994), los pastizales representan la comunidad de mayor dispersión y cobertura dentro del paisaje de suelos arenosos pardo - amarillento y planosoles, cubriendo aproximadamente un $70 \%$ de la superficie.

Es importante señalar que si bien el fuego es una herramienta de uso frecuente, no hay registros bibliográficos suficientes; o son muy escasos en nuestro país del efecto sobre los pastizales. En lo 
que respecta a la acción que ejerce sobre la fauna de invertebrados que han colonizado esos pastizales, no hemos hallados en el registro bibliográfico trabajos previos en nuestro país, si a nivel internacional que sirvieron de base para el presente trabajo.

Los estudios del comportamiento de las poblaciones de insectos y de los factores ambientales que las afectan, no tienen únicamente interés ecológico, sino también son importantes para comprender y controlar aquellos grupos taxonómicos de insectos de incidencia negativa y/o positiva en el desarrollo de los vegetales (Pianka, 1982). Además en la dinámica poblacional, resulta de interés determinar la influencia de los factores abióticos en el ciclo biológico de cada grupo de insectos en las diferentes estaciones del año.

El efecto del fuego sobre el suelo depende de la intensidad del mismo y las características del suelo, pero generalmente altera su permeabilidad (Debano et al, 1979). La fauna sufre también los efectos del fuego, que a corto plazo puede matar individuos por calor o asfixia sin afectar de manera apreciable a las poblaciones y comunidades, las que si son afectadas a largo plazo por el cambio de hábitat (GarcíaVillanueva, 1992).

Dentro de los invertebrados, los anélidos (incluye a las lombrices de tierra) representan a los grupos más afectados por las condiciones de sequía del suelo, así como por las nuevas condiciones fisicoquímicas creadas por la acción del fuego y la destrucción de la materia orgánica (Curry, 1987; Heyward y Tissot, 1936; Philips, 1965).

Bajo el supuesto de que la quema actúa de manera diferencial sobre los diferentes órdenes de invertebrados, el objetivo de este trabajo ha sido estudiar el efecto del fuego sobre los diferentes órdenes de insectos presentes en un pastizal, con predominio de Andropogon lateralis y Sorghastrum agrostoides, en el NO de la provincia de Corrientes, Argentina, a través de relevamientos pre y post quema de la fauna de invertebrados presente en el área de estudio.

\section{MATERIALES Y METODOS}

El trabajo de campo se realizó, entre 1998 y 2000, en un pastizal situado en la EEA INTA Corrientes dentro del área ecológica del Albardón del Río Paraná, sometido a tres frecuencias de quema. Los tratamientos aplicados al pastizal fueron: $\mathrm{T}_{0}=\sin$ quema, $\mathrm{T}_{1}=$ quema anual (septiembre de 1998, agosto de 1999) y $\mathrm{T}_{2}=$ quema bianual (septiembre de 1998), en un diseño de bloques completos al azar con tres repeticiones. El material combustible existente en el momento de aplicar los tratamientos consistió en una biomasa con el $80 \%$ de material muerto en pie.

Se realizaron relevamientos de la fauna aérea y de suelo pre y postquema (un mínimo de 45 días después de la quema) en: mayo, noviembre y diciembre de 1998, marzo, mayo, julio, septiembre, noviembre y diciembre de 1999, marzo y mayo de 2000.

La fauna aérea se relevó mediante redes entomológicas de malla fina y aspiradores, el tiempo de recolección fue de 20 minutos, el material obtenido se colocó en frascos de vidrio preparados ad hoc, para su posterior determinación sistemática mediante el uso de claves dicotómicas. La presencia de fitófagos sobre la flora, se detectó mediante observaciones visuales, examinando los órganos aéreos (tallos, hojas etc.), registrando los insectos hallados.

Para el estudio de la fauna del suelo, en cada parcela, se recogieron muestras a $20 \mathrm{~cm}$ de profundidad en bolsas de nylon. Posteriormente al secado de las muestras, fueron pasadas por cedazo de malla fina-intermedia, para separar los ejemplares bajo microscopio estereoscópico, clasificarlos y conservarlos en cámaras de algodón y/o frascos de vidrio con alcohol 70\%.

Con la información obtenida se efectuó análisis multivariante de conglomerados jerárquicos con el fin de evaluar el comportamiento de la fauna estudiada por oportunidad de muestreo y el análisis de la variancia por grupo taxonómico, según el diseño estadístico utilizado, y la prueba de Tukey para separación de medias $(\alpha=0,05)$. Utilizando el software estadístico SPSS y Statgraphics.

\section{RESULTADOS}

Tanto en los muestreos de prequema como en los de postquema, los grupos taxonómicos encontrados fueron: Phylum Arthropoda, Clase Insecta, con los siguientes Órdenes: Hymenóptera, Hemíptera, Coleóptera, Lepidóptera, Odonata, Díptera, Ortóptera y Mantódea; Clase Arachnida, con los Órdenes Aranea y Acarina; Clase Crustacea, Orden Isópoda; Clase Miriápoda, Orden Julifornia; Phylum Nemátoda; Phylum Annélida, Clase Oligochaeta.

De los grupos taxonómicos encontrados, las variaciones entre la prequema y la postquema que se observan son: incremento en la cantidad y variabilidad de Díptera, (DIP), Lepidóptera (LEP) y Coleóptera (COL); incremento en la cantidad y disminución de la variabilidad de Hemíptera (HEM), Mantódea (MAN), Ortóptera (ORT), Hymenóptera (HYM), Odonata (ODO) y Arácnida (ARA) (Figuras $1 \quad$ y $\quad 2$ ). 


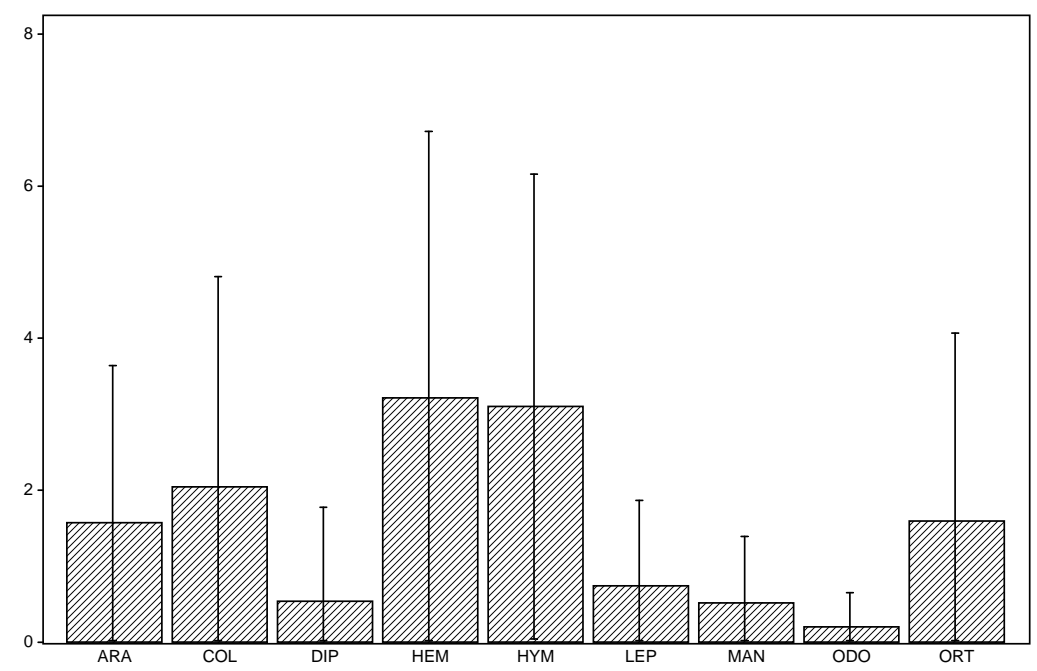

Figura 1: Promedio y desviación estándar del número de invertebrados de los diferentes órdenes o clases encontrados por parcela. Muestreos pre-quema.

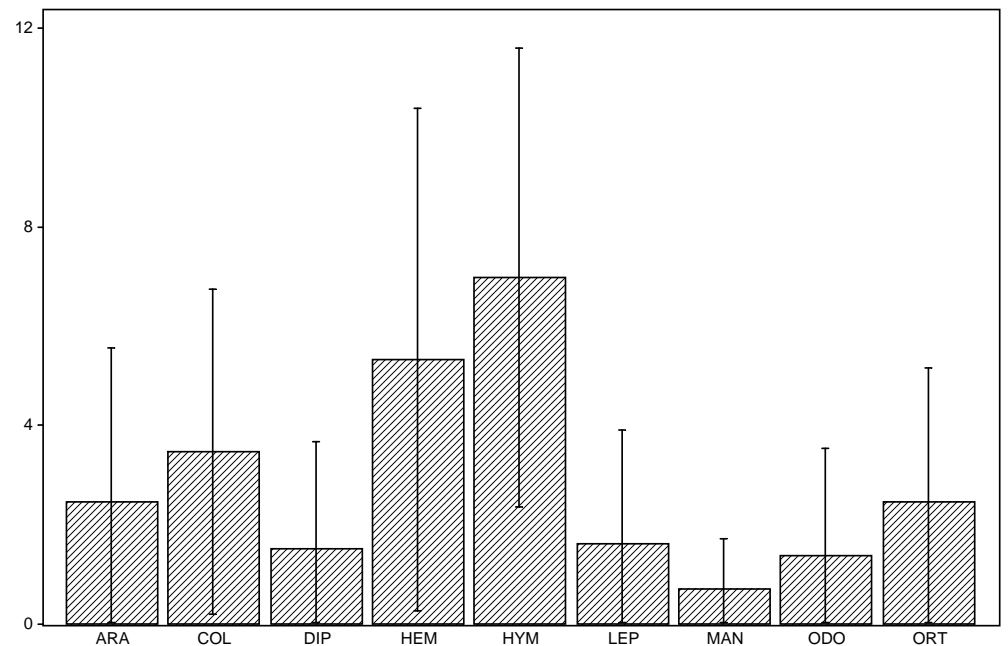

Figura 2: Promedio y desviación estándar del número de invertebrados de los diferentes órdenes o clases encontrados por parcela. Muestreos post-quema.

El análisis de conglomerados realizado por parcela y momento ha arrojado el siguiente dendrograma (Figura 3). Esto estaría indicando que en el comportamiento de los diferentes grupos taxonómicos inciden más las características del lugar o las condiciones del clima que la quema únicamente. Con respecto a los agrupamientos que se han logrado por similitudes en el comportamiento, se puede conformar seis conglomerados, el primero formado por Hemíptera, Coleóptera, Miriápoda, Acarina y Oligaqueta; el segundo por Díptera y Aranea; el tercero por Lepidóptera, Odonata y
Ortóptera; el cuarto por Mantódea e Isópoda; el quinto por Hymenóptera y el sexto por Nemátoda.

En lo que respecta a los tratamientos aplicados, a través del análisis de la variancia y posterior prueba de Tukey se determinó en T2= quema bianual una menor cantidad de individuos de las categorías taxonómicas: Hemíptera, Coleóptera, Lepidóptero, Mantódea que en T1= quema anual y menor cantidad de Aránea, Isópoda, Miriápoda, Acarina y Oligaqueta que en T1= quema anual y $\mathrm{T} 0=\sin$ quema. 


\section{Encadenamiento promedio (average linkage)}

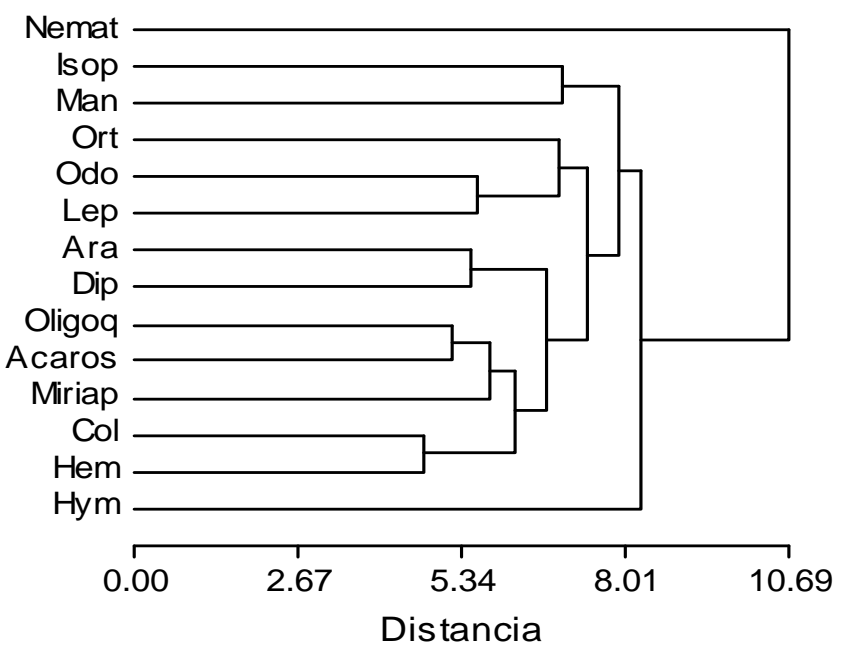

Figura 3. Resultado del análisis de conglomerados jerárquicos por parcela.

\section{DISCUSION}

En la composición de la población de los diferentes grupos de invertebrados tanto de suelo como aérea, se observaron variaciones entre los diferentes muestreos, (Pre y postquema) posiblemente esto estaría asociado no solo al factor quema solamente sin también a un factor estacional.

Coincidentemente con las observaciones realizadas por (Campalans, 1984), el incendio favorece el desarrollo de los insectos de menor talla, esto lo observamos respecto al grupo de los Hymenópteros a la vez que disminuye la diversidad específica.

En cuanto a los Coleópteros se observó una mayor cantidad y variabilidad en la población después de la quema en las estaciones cálidas, al respecto French \& Keirle (1969) encontraron los siguientes grupos de Coleópteros (Carábidos y Escarabeidos) inmediatamente después del fuego, mientras Buffington (1967) encontró cuatro veces menos coleópteros en áreas quemadas, aunque pareciera que tras una reducción inicial, recolonizan rápidamente (Rice, 1933). También se ha observado una presencia diferencial en la población de elatéridos entre áreas quemadas y no quemadas en Bielorusia (Rubzovca, 1967).

Los Miríapodos sufrieron también una disminución en la cantidad de individuos recolectados, según Pradon et al (1987), la población de Miriápodos resulta afectada según el grupo, así los más afectados son los Júlidos que se encuentran en los primeros $20 \mathrm{~cm}$ de profundidad del suelo.

El rebrote temprano y vigoroso de la vegetación herbácea, alimenta a una fauna más densa de invertebrados herbívoros durante la estación propicia (Andrzejewska 1979; Guillon, 1971). Los ortópteros serán uno de los grupos más afectados por esta circunstancia, aunque parece ser que el fuego no genera patrones regulares de cambio demográfico en las poblaciones locales de saltamontes (Evans, 1988), aunque fuegos periódicos podrían servir como mecanismo imperfecto de reajuste (Cummins, 1979; Fisher et al., 1982).

\section{CONCLUSIONES}

Los diferentes grupos de invertebrados hallados en la comunidad vegetal objeto de estudio fueron afectados de diferente manera por la quema: Los ordenes Díptera, (DIP), Lepidóptera (LEP) y Coleóptera (COL), han incrementado su población en cantidad y variabilidad, lo cual indicaría un efecto beneficioso de la quema. En tanto que los ordenes Hemíptera (HEM), Mantódea (MAN), Ortóptera (ORT), Hymenóptera (HYM) y Odonata (ODO) si bien se registra un aumento en la cantidad se observa una disminución de la variabilidad. Esto nos indicaría un efecto perjudicial del fuego. Las diferencias observadas en el comportamiento de la fauna de invertebrados en la comunidad de vegetal estudiada, estaría dada no solamente por el factor quema sino también relacionada directamente con las estaciones principalmente durante la primavera - verano, época de eclosión de las oviposiciones y desarrollo de los estadios inmaduros, pues por tratarse de animales heterotermos con bajas temperaturas generalmente se encuentran en letargo entre la hojarasca o a escasos centímetros de la superficie del suelo. 


\section{BIBLIOGRAFIA}

Andrzejewska, L. 1979. Herbivorous fauna and mammals. En Fire and Ecosystems. L herbivorous in natural and managed meadows. Polish Ecological Studies 5. 544.

Buffington, J.D. 1967. Soil arthropod populations of the New Jersey pine barrens as affected by fire. Ann. Entomol. Soc. Amer. 60: 530-535.

Campalans, J. 1984. Efecto del fuego sobre la taxocenosis coleopterológica epígea del matorral nativo de la V Region. Tesis de licenciatura. Univ. Católica de Valparaíso. P. 150.

Carnevali, R. 1994. Fitogeografía de la Provincia de Corrientes. 324p.

Cummins, K.W. 1979. The natural stream ecosystem. In Ward, J.V. and Stanford, J.A. (Eds). The ecology of regulated streams, Plenum Press, New York. 7 - 24.

Curry, J.P. 1987. The invertebrate fauna of grassland and its influence on productivity. II. Factors affecting the abundance and composition of the fauna. Grass and Forage Science 42. 197-212.

Debano, L.F.; R.M. Rice y C.E. Conrad. 1979. Soil heating in chaparral fires: effects on soil properties, pllant nutrients, erosion and runoff, Res. Paper. Pac. Southwest For, Range Exp. Stn. PSW- 145.

Evans, E.W. 1988. Community dynamics of prairie grasshoppers subjected to periodic fire: predictable trajectories or random walks in time? Oilos 52. 283-292.

Fisher, S.G.; L.J. Gray; N.B. Grimm y D.E. Busch. 1982. Temporal seccession in desert stream ecosystem following flash flooding. Ecol. Monogr. 52. 93-110.

French, J.R.S. \& R.M. Keirle 1969. Studies in fire damaged radiata pine plantations. Aust. Forest. 33. 175-180.
García Villanueva, J.A. 1992. Recolonización por artrópodos de zonas alteradas por el fuego y otros procesos degradativos. Tesis doctoral. Área ecología Facultad de Biología. Universidad de León (España) Pág. 150.

Guillon, Y. 1971. The effect of bush fire on the principal Acridid species of an Coast savanna. En: Proceedings of the Annual Tall Timers Fire Ecology Conference $N^{\circ}$ 11. 419-471.

Heyward, F. \& A.N. Tissot 1936. Some changes on soil fauna asociated with forest fires in the long leaf pine region. Ecology 17. 659-666

Mooney, H.A. y R.H. Hobbs. 1986. Resilience at the individual plant level. In: Dell, D., Hopkins, A.J.; Lamont, B.B. (eds) Resilience in Mediterrenean type ecosystems. P. 65-82. La Haya: Dr W. Junk.

Phillips, J. 1965. Fire as master and servant. It“s influence on the bioclimatic regions of trans-Sahara Africa. Tall Timbers Fire Ecol. Conf. Proc. 4: 7-109.

Pianka, E.R. 1982. Ecología Evolutiva. Omega. Pp.220. S. A Barcelona

Pradón, R.; R. Fons y F. Athias-Binche 1987. The impact of fire on animal communities in mediterranean area. In: The role of fire in ecological systems. Ed. L. Trabaud. S.P.B. Academec Publishing 121-157. The Hague.

Rice, I. 1933. The effect of fire on praire animal communities. Ecology. 13: 392-401.

Rubzovca, Z.I. 1967. Elateridae in soil of pine forest types in west Bielorussia. Oikos, 18. 41-54.

Tárrega, R. 1986. Regeneración post-fuego del estrato herbáceo en robledales de Quercus pyrenaica en la provincia de León. Tesis doctoral. Univ. De León. P.110

Vera, M.L.; M.C. Alonso y J.R. Obeso 1995. Rebrote después de incendio e inversión reproductiva en Erica cinerea y E. Vagans. Orsis 10, 7-15. 